\title{
Video Article \\ Multi-electrode Array Recordings of Human Epileptic Postoperative Cortical Tissue
}

\author{
Elena Dossi ${ }^{1}$, Thomas Blauwblomme ${ }^{2,3}$, Rima Nabbout ${ }^{2,4}$, Gilles Huberfeld ${ }^{2,5}$, Nathalie Rouach ${ }^{1}$ \\ ${ }^{1}$ Neuroglial Interactions in Cerebral Physiopathology, Center for Interdisciplinary Research in Biology, CNRS UMR 7241, INSERM U1050, Collège de France \\ ${ }^{2}$ Infantile Epilepsies \& Brain Plasticity, INSERM U1129, PRES, Paris Descartes University, Sorbonne Paris Cité, CEA \\ ${ }^{3}$ Neurosurgery Department, Necker Hospital, AP-HP, Paris Descartes University \\ ${ }^{4}$ Rare Epilepsies Reference Center, Necker Hospital, AP-HP, Paris Descartes University \\ ${ }^{5}$ Neurophysiology Department, La Pitié-Salpêtrière Hospital, AP-HP, Sorbonne and Pierre and Marie Curie University
}

Correspondence to: Nathalie Rouach at nathalie.rouach@college-de-france.fr

URL: https://www.jove.com/video/51870

DOI: doi: $10.3791 / 51870$

Keywords: Medicine, Issue 92, electrophysiology, multi-electrode array, human tissue, slice, epilepsy, neocortex

Date Published: 10/26/2014

Citation: Dossi, E., Blauwblomme, T., Nabbout, R., Huberfeld, G., Rouach, N. Multi-electrode Array Recordings of Human Epileptic Postoperative Cortical Tissue. J. Vis. Exp. (92), e51870, doi:10.3791/51870 (2014).

\section{Abstract}

Epilepsy, affecting about $1 \%$ of the population, comprises a group of neurological disorders characterized by the periodic occurrence of seizures, which disrupt normal brain function. Despite treatment with currently available antiepileptic drugs targeting neuronal functions, one third of patients with epilepsy are pharmacoresistant. In this condition, surgical resection of the brain area generating seizures remains the only alternative treatment. Studying human epileptic tissues has contributed to understand new epileptogenic mechanisms during the last 10 years. Indeed, these tissues generate spontaneous interictal epileptic discharges as well as pharmacologically-induced ictal events which can be recorded with classical electrophysiology techniques. Remarkably, multi-electrode arrays (MEAs), which are microfabricated devices embedding an array of spatially arranged microelectrodes, provide the unique opportunity to simultaneously stimulate and record field potentials, as well as action potentials of multiple neurons from different areas of the tissue. Thus MEAs recordings offer an excellent approach to study the spatiotemporal patterns of spontaneous interictal and evoked seizure-like events and the mechanisms underlying seizure onset and propagation. Here we describe how to prepare human cortical slices from surgically resected tissue and to record with MEAs interictal and ictal-like events ex vivo.

\section{Video Link}

The video component of this article can be found at https://www.jove.com/video/51870/

\section{Introduction}

Epilepsy is a chronic disorder in which epileptic seizures, which are intermittent patterned discharges lasting several seconds to tens of seconds on electroencephalographic (EEG) recordings associated with clinical manifestations, interrupt an interictal state, characterized by the presence of synchronous neuronal discharges lasting tens of milliseconds and called interictal events ${ }^{1}$. It affects approximately $1 \%$ world's population and although seizures are controlled in the majority of the patients, approximately one third of people with epilepsy do not show adequate response to antiepileptic drugs ${ }^{2}$. In this condition, called pharmacoresistant epilepsy and whose mechanisms still have to be clearly identified, the surgical resection of the specific part of the brain identified as the seizure-onset zone remains the only alternative treatment giving a positive outcome to patients. Thus, the resected specimens from surgery yield the opportunity to study the mechanisms of interictal discharges and seizure generation and propagation, as well as pharmacoresistance on viable human central nervous tissue ex vivo.

Multi-electrode arrays (MEAs), consisting of an arrangement of spatially distributed microelectrodes, allow the simultaneous stimulation and recording of electrophysiological activity from several sites of the tissue, thus providing an excellent approach to study the spatio-temporal patterns of spontaneous and evoked activity. This technique, first applied to monitor the developmental changes of neuronal cell culture activity ${ }^{3}$ and then adapted for acute and organotypic brain and spinal cord slices ${ }^{4-6}$, is presently considered a valuable electrophysiological tool.

The current protocol describes how to prepare human cortical slices from surgically resected tissue and to record with MEAs reliable ex vivo interictal and seizure-like events from these slices. This technique thus provides a way to address the basic mechanisms underlying epileptic activities initiation, propagation and effects of antiepileptic drugs at both the cellular and network levels. All the procedures used to obtain, prepare, maintain and record human slices are here described in detail.

\section{Protocol}

NOTE: The protocol here described follows the guidelines of the French "Comité Consultatif National d'Ethique" and is declared as a collection activity by INSERM. 


\section{Resection of Human Epileptic Tissue}

1. Patients

1. Obtain brain tissue from patients suffering from intractable epilepsy. Obtain a written informed consent before surgery.

2. For all operated epileptic patients perform an extensive presurgical workup including neurological examination, neuropsychological assessment, surface EEG, and neuroimaging (MRI and sometimes ${ }^{18} \mathrm{FDG}-\mathrm{PET}$ scan; Figure 1A), followed by post-surgical histological examination of resected tissue (Figure 1B). NOTE: Surgery is indicated when the diverse explorations localize similarly the seizure onset zone and when the benefit of its removal exceeds the surgical risks.

2. Surgery

1. Maintain antiepileptic drugs pre-operatively. Perform surgery under general anesthesia: induction with inhaled sevoflurane $(6 \%$ delivered ratio and $100 \%$ inhaled fraction of $\left.\mathrm{O}_{2}\right)$, intra venous sufentanyl $(0.3 \mu \mathrm{g} / \mathrm{kg})$ and antracurium $(0.5 \mathrm{mg} / \mathrm{kg})$, followed by maintenance with intra venous sufentanyl $(0.5 \mu \mathrm{g} / \mathrm{kg} / \mathrm{hr})$ and inhaled sevoflurane (1 MAC).

2. Use a neuronavigation system to allow a precise localization of the lesioned cortex (Figure 1C). After skin incision, perform a burr hole in the bone followed by a craniotomy with a high spill drill. Gently elevate the bone flap, and open the dura with scissors (Figure 1D).

3. Use intra-operative ultra sound to facilitate the identification of the abnormal cortex, and microsurgical instruments and an ultrasonic aspirator under the operating microscope.

4. Coagulate and cut the pio-arachnoidal plane according to the sulcal limits. Use subpial dissection to release the cortex from the pia around the lesioned area.

5. Cut the white matter under the abnormal cortex to perform one piece "en bloc" resection, sparing as much as possible the vasculature. Avoid traumatic manipulation of the cortex.

6. Cut a $1 \mathrm{~cm}$ thick slice out of the resected tissue along a direction orthogonal to the pial surface, including the sulcus bottom and transitional cortex.

\section{Artificial Cerebrospinal Fluid (ACSF) for Slice Preparation, Incubation and Recording}

1. ACSF for slice preparation

1. Prepare $1 \mathrm{~L}$ of sucrose-based $\mathrm{ACSF}^{7,8}$, containing (in $\mathrm{mM}$ ): 250 sucrose, $3 \mathrm{KCl}, 25 \mathrm{NaHCO}_{3}, 10 \mathrm{D}-\mathrm{Glucose}_{1}, 1 \mathrm{CaCl}_{2}, 10 \mathrm{MgCl}_{2}$; dissolve these salts in deionized water and oxygenate the solution with carbogen for at least $10 \mathrm{~min}$.

2. Put $\sim 700 \mathrm{ml}$ of sucrose ACSF at $-80^{\circ} \mathrm{C}$ for $50-60 \mathrm{~min}$ (or $-150^{\circ} \mathrm{C}$ for $15-20 \mathrm{~min}$ ). Keep the rest of the solution under oxygenation in ice. NOTE: The ACSF for slice preparation can be stored at $4{ }^{\circ} \mathrm{C}$; in this case, add $\mathrm{CaCl}_{2}$ and $\mathrm{MgCl}_{2}$ the day of the experiment, before cooling it.

2. ACSF for slice incubation and recording

1. Prepare at least $3 \mathrm{~L}$ of recording $\mathrm{ACSF}^{7,8}$, containing (in $\mathrm{mM}$ ): $124 \mathrm{NaCl}, 3 \mathrm{KCl}, 26 \mathrm{NaHCO}_{3}, 10 \mathrm{D}-\mathrm{Glucose}_{1}, 1.6 \mathrm{CaCl}_{2}, 1.3 \mathrm{MgCl}_{2}$; dissolve these salts in deionized water and oxygenate the solution with carbogen. Before adding $\mathrm{MgCl}_{2}$, keep some ACSF to perform recordings in $0 \mathrm{Mg}^{2+}$ ACSF. NOTE: The incubation/recording ACSF can be stored at $4{ }^{\circ} \mathrm{C}$; in this case, add $\mathrm{CaCl}_{2}$ and $\mathrm{MgCl}_{2}$ the day of the experiment.

\section{Equipment for Slice Incubation and Recording}

1. Interface chamber for slice incubation

1. Use a brain slice chamber to maintain living slices ex vivo in interface mode. NOTE: The chamber is composed of a lower section, which contains the heating and the sensor elements and a ceramic bubbler and is filled with distilled water, and an upper part, where the slices rest on a sheet of lens tissue in the flat area in the center of the chamber (Figure 2A-B).

2. Use two separate fine PTFE tubes, through which the preoxygenated ACSF enters the chamber. NOTE: The tubes spiral in the heated water and reach the upper part of the chamber; then the solution exits by means of capillary action with lens tissue, that conveys the solution into exit wells.

3. Connect the PTFE tubes of the interface chamber to a peristaltic pump, to allow continuous perfusion of the slices with the preoxygenated medium.

4. Maintain the high oxygen tension by oxygenating with carbogen $\left(95 \% \mathrm{O}_{2}\right.$ and $\left.5 \% \mathrm{CO}_{2}\right)$ through the ceramic bubbler. NOTE: This warmed and moistened gas mixture reaches the slices in the upper part of the chamber through appropriate holes.

5. Maintain the temperature in the upper part of the chamber by connecting the chamber to a temperature controller, through a special double-ended cable, having a connector for the chamber heating element and an external temperature sensor at one end. Place the sensor near the slices to check the temperature throughout the incubation (Figure 2C).

2. Multi-electrode array recordings

1. Use planar multi-electrode arrays with 120 titanium nitride electrodes (30 $\mu \mathrm{m}$ diameter) insulated with silicon nitride and internal reference electrode, arranged in a $12 \times 12$ matrix with $200 \mu \mathrm{m}$ center-to-center spacing. NOTE: Other configurations with different electrode diameter and spacing are possible. In particular, the MEA chip can be widened to sample larger slices of human tissues.

2. Acquire electrical signals at $10 \mathrm{kHz}$ using a MEA2100-120 system (pre- and filter amplifier with integrated data acquisition and analogdigital converter, 16 bit data resolution, input voltage range and bandwidth adjustable via software) through a dedicated software (MC_Rack). NOTE: The MEA2100 headstage is endowed of a metal plate for fixing of heatable perfusion element through magnetic holders, to continuously perfuse the slices throughout the recording session. 
3. Hold the tissue down on the MEA by a small homemade platinum anchor, which ensures a good electrical coupling between the slice and the electrodes.

\section{Preparation of Interface Chamber and Tools for Dissection and Slicing}

1. Interface chamber

1. Fill the lower section of the interface chamber with distilled water. Ensure that the level of water completely immerses the heating element and is 2 to $4 \mathrm{~mm}$ below the junction between the lower and upper section of the chamber. Check this level daily before switching on the heating, in order to avoid damage of the heating element.

2. Connect the chamber to a carbogen source, endowed with a secondary flow regulator for fine adjustments of gas pressure.

3. Cut a sheet of lens tissue in order to fit the flat area of the slice chamber, and position it close to input solution tubes, to let any bubble escape from the input line before reaching the slices.

4. Cut two pieces of stranded cotton thread as long as the piece of lens tissue, and position them along the main sides of the flat area of the chamber. NOTE: This helps to slightly raise the perfusion solution level in the chamber.

5. Set the flow rate of the peristaltic pump at $1 \mathrm{ml} / \mathrm{min}$ and activate the pump.

6. Start oxygenation of the water in the bottom part of the chamber.

7. Set the temperature of the temperature controller at $37^{\circ} \mathrm{C}$ and turn it on. Cover the upper part of the interface chamber with a piece of parafilm and wait at least $30 \mathrm{~min}$ for the temperature to increase and stabilize.

2. Tools for dissection and slicing

1. Prepare a dissection kit consisting of two fine forceps, a spatula, a small spoon and a blade; two glass petri dishes, two brushes as well as cyanoacrylate glue.

2. Set the parameters of the vibratome: thickness, $400 \mu \mathrm{m}$; frequency, $70-90 \mathrm{~Hz}$; amplitude, $0.9-1 \mathrm{~mm}, \mathrm{speed}: 0.1 \mathrm{~mm} / \mathrm{sec}$.

\section{Human Cortical Slice Preparation}

1. Remove the sucrose-based ACSF from $-80^{\circ} \mathrm{C}$ freezer and mix it in order to obtain a sort of slush. Oxygenate it with carbogen, put $\sim 250 \mathrm{ml}$ in a glass bottle and keep it in a Dewar bottle filled with ice, while going to the surgery room of the hospital to get the tissue. In the meantime, keep the rest at $-20^{\circ} \mathrm{C}$.

NOTE: During the surgery, the neurosurgeon dissects a small block of cortical tissue. Immediately place the sample in ice-cold sucrose ACSF for transfer to the laboratory.

2. Keep the time needed for the transfer from the hospital to the laboratory to a maximum of $30 \mathrm{~min}$. In the laboratory, take the sucrose-based oxygenated ACSF out of $-20^{\circ} \mathrm{C}$ freezer, mix it to have a slush, fill a petri dish and the vibratome buffer tray and start oxygenating both with carbogen. Carefully take out the piece of human tissue from the bottle with a spoon and put it in the petri dish (Figure 2D).

3. Using the forceps, carefully remove blood clots, vessels and meninges to avoid resistance during slicing. Remove the meninges, consisting in pia-matter, starting from the sides of the tissue block, paying attention not to damage the cortical surface. With a blade, cut a side of the tissue to obtain a flat surface, which will be glued on the vibratome specimen plate. NOTE: The meninges, consisting in pia-matter, are removed starting from the sides of the tissue block, paying attention not to damage the cortical surface.

4. If the block of tissue is bigger than the MEA chamber, cut a piece of appropriate dimensions before gluing it on the specimen plate (Figure 2E).

5. Glue the tissue in order to obtain transverse cortical slices, put it into the buffer tray and cut $400 \mu \mathrm{m}$ thick slices at low speed $(0.1 \mathrm{~mm} / \mathrm{sec})$ (Figure 2F).

6. Cut pieces of lens tissue with slice dimensions; with a spatula and a brush, transfer the slices on these lens tissues and incubate them in the interface chamber at $37^{\circ} \mathrm{C}$ for at least $1 \mathrm{hr}$ before starting recordings (Figure 2G-I). Continuously store the slices in the same conditions until use. NOTE: Placing slices on lens paper is an important step to facilitate both the perfusion of bottom side of slices and the removal of slices from the interface chamber before recording.

1. While transferring the slices on the lens tissue, manage them very carefully, avoiding to touch the cortical layer area with the brush.

2. Prepare the slices one at a time and immediately transfer each of them to the interface chamber, in order to ensure proper oxygen supply and temperature; slice the block of resected tissue as quickly as possible.

\section{Preparation of MEA Setup for Recordings}

1. Plug the perfusion system to a peristaltic pump and the MEA system to a computer. Oxygenate the recording ACSF. Place an empty MEA chip inside the amplifier.

2. Set the temperature controller for heating cannula element to reach $37^{\circ} \mathrm{C}$ in the MEA chamber and start the perfusion at $5-6 \mathrm{ml} / \mathrm{min}$. NOTE: It is usually necessary to set the temperature $3-4{ }^{\circ} \mathrm{C}$ higher than the one desired, depending on the room temperature and the flow rate.

1. Check the temperature in the MEA chamber with a fine thermometer, placing it the closest possible to the center, without touching the electrode area.

3. Start recording software and check that all the MEA electrodes are well connected and that there is no noise due to perfusion.

4. Start MEA_Monitor to check the functioning of the camera table. 


\section{Transfer of a Slice From Interface Chamber to MEA Chip for Recording}

1. Pour some warmed recording ACSF in a glass Petri dish; with a forceps, take a slice from the interface chamber, by grabbing it through the lens tissue, and put it in the petri dish. Carefully detach the slice from the tissue. If the level of the solution in the interface chamber is carefully adjusted during incubation time, the slice will detach from the lens tissue only by submerging it in the solution; otherwise, use a small brush to facilitate the detachment.

2. Fill a MEA chip with some ACSF and with a spatula, transfer the slice into it. With a plastic Pasteur pipette, gently remove the solution to make the slice adhere on the electrode area and keep it in place with a platinum anchor. Add $1 \mathrm{ml}$ of recording ACSF, place the MEA chip into the amplifier and start perfusion at 5-6 $\mathrm{ml} / \mathrm{min}$ (Figure $2 \mathrm{~J}-\mathrm{L}$ ).

3. Check the slice position on the MEA recording area by using MEA Monitor, adjust it if necessary in order to record from the cortical layers and take a picture. Start the recording.

\section{Representative Results}

The recording of human cortical slice activity starts while perfusing the tissue with normal recording ACSF (as previously described) $)^{7,8}$. In this condition, when the tissue is well preserved during surgery and later on during tissue transfer from the hospital and slice preparation, one can observe spontaneous activity, in the form of small interictal-like events, detected from small clusters of MEA electrodes. Interictal-like discharges consisted in a field potential, usually biphasic, comprising an initial sharp negative deflection, reaching tens of microvolts, followed by a longer opposite wave lasting several tens of milliseconds. Fast multi-unit activities are usually embedded in the field potential mainly in the initial part. A representative example of interictal-like activity is illustrated in Figure 3Aii, where the trace recorded by a MEA electrode is shown.

To record epileptic seizures from in vitro human cortical slices, it is necessary to perfuse them with an "epileptogenic" ACSF, in which Mg ${ }^{2+}$ is absent and $\mathrm{K}^{+}$is raised to $6 \mathrm{mM}$, to boost tissue excitability ${ }^{1}$ (the increase of $\mathrm{K}^{+}$from 3 to $6 \mathrm{mM}$ alone is not enough to induce seizures; data not shown). Once the perfusion with $0 \mathrm{Mg}^{2+} 6 \mathrm{mM} \mathrm{K}^{+} \mathrm{ACSF}$ has started, the activity of cortical slices gradually increases and the first seizure appears within 15 to $20 \mathrm{~min}(15.4 \pm 1.7 \mathrm{~min}, n=10$, from 5 patients; Figure $3 \mathbf{A i}$ ). We evoked ictal-like activity, as shown in Figure 3 , in $75 \%$ of patients tested and in $66.7 \%$ of all recorded slices.

Epileptic activities are recorded from adjacent electrodes of the MEA chip, and comparison of the onset dynamics of field potential events allows studying their site of genesis and propagation. A representative seizure recorded from a slice of human cerebral cortex with the MEA system is shown in Figure 3B (a representative trace of a seizure recorded by a single MEA electrode is shown at higher temporal resolution in Figure 3 Aiii). Note that the seizure is recorded from almost all the electrodes of the MEA chip, thus indicating that it is able to propagate to a large cortical area. Furthermore, looking at the single traces recorded from each electrode, it is possible to observe the progressive propagation of the seizure across the tissue: indeed, it starts first in the cortical area covering the right half of the electrode array, and it gradually spreads to the area covering the left half (i. e., compare traces from electrode L6 and B6; Figure 3B). 

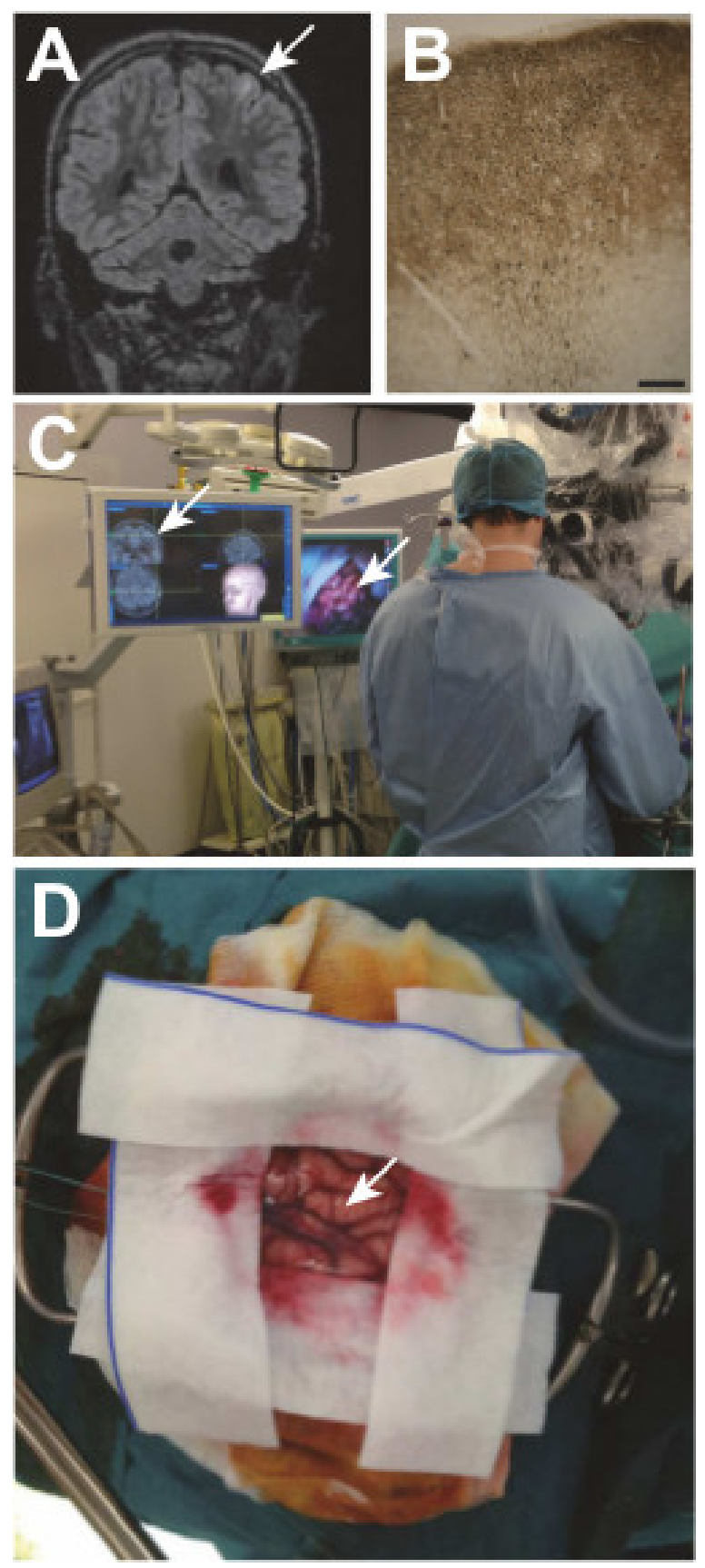

Figure 1: A cortical dysplasia and its surgical removal. A Taylor type focal cortical dysplasia (white arrow) embedded in a left frontal lobe sulcus is visualized on the preoperative MRI (A), and later confirmed by histological examination (B) MAP kinase labeling; scale bar: $200 \mu \mathrm{m}$ ) showing a cortical dyslamination, abnormal neurons and a track on neurons with an incomplete migration in the lower white matter. During surgery, the dysplasia is localized according to the MRI data with a neuronavigation system (C). Visualization of the gyrus containing the dysplasia during surgery (D). 

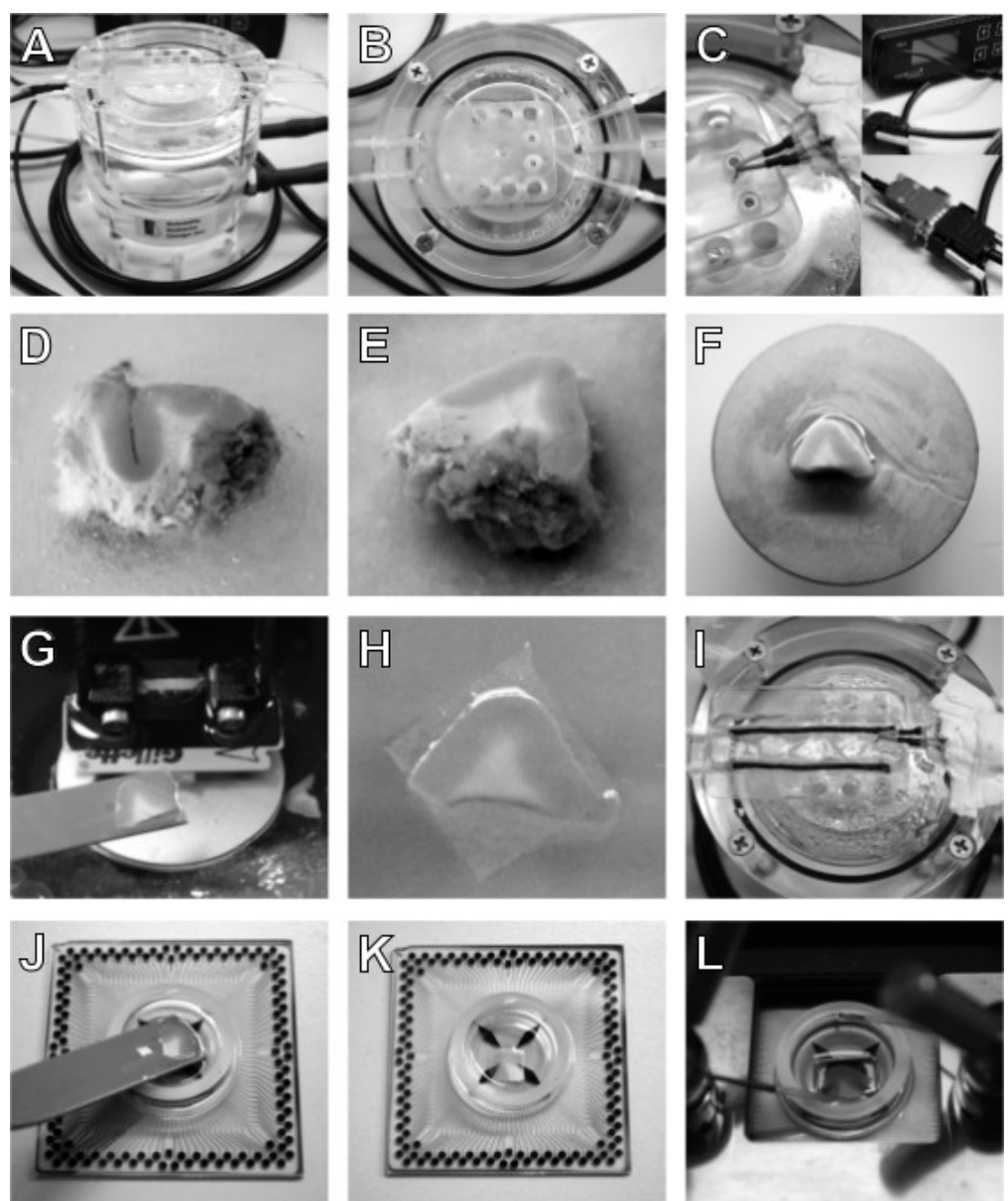

Figure 2: Equipment and procedure for human cortical slice preparation. An interface chamber is used to maintain human cortical slices ex vivo (A); the slices rest on a sheet of lens tissue in the flat area in the upper part of the chamber (B), where a temperature sensor (C, left), connected to a temperature controller (C, right, up) through a double-ended cable (C, right, down), is placed to maintain $37^{\circ} \mathrm{C}$ throughout slice incubation time. Once obtained, place the resected block of tissue in a petri dish filled with ice-cold sucrose-based ACSF (D) and remove blood clots, vessels and meninges, to facilitate the slicing. If the tissue block is bigger than MEA chambers, isolate a piece of appropriate dimensions with a blade (E) and glue it on the vibratome specimen plate (F). Cut $400 \mu \mathrm{m}$ thick slices and with the help of a spatula (G) transfer them on pieces of lens tissue $(\mathbf{H})$ and incubate them in the interface chamber (I). Before recording, remove the lens tissue by submerging the slice in a petri dish filled with ACSF and transfer it to an ACSF filled MEA chip with a spatula (J). Remove the solution to let the slice adhere on the MEA (K) and keep it in position by using a platinum anchor. Place the MEA in the amplifier (L), start perfusion and recording. 

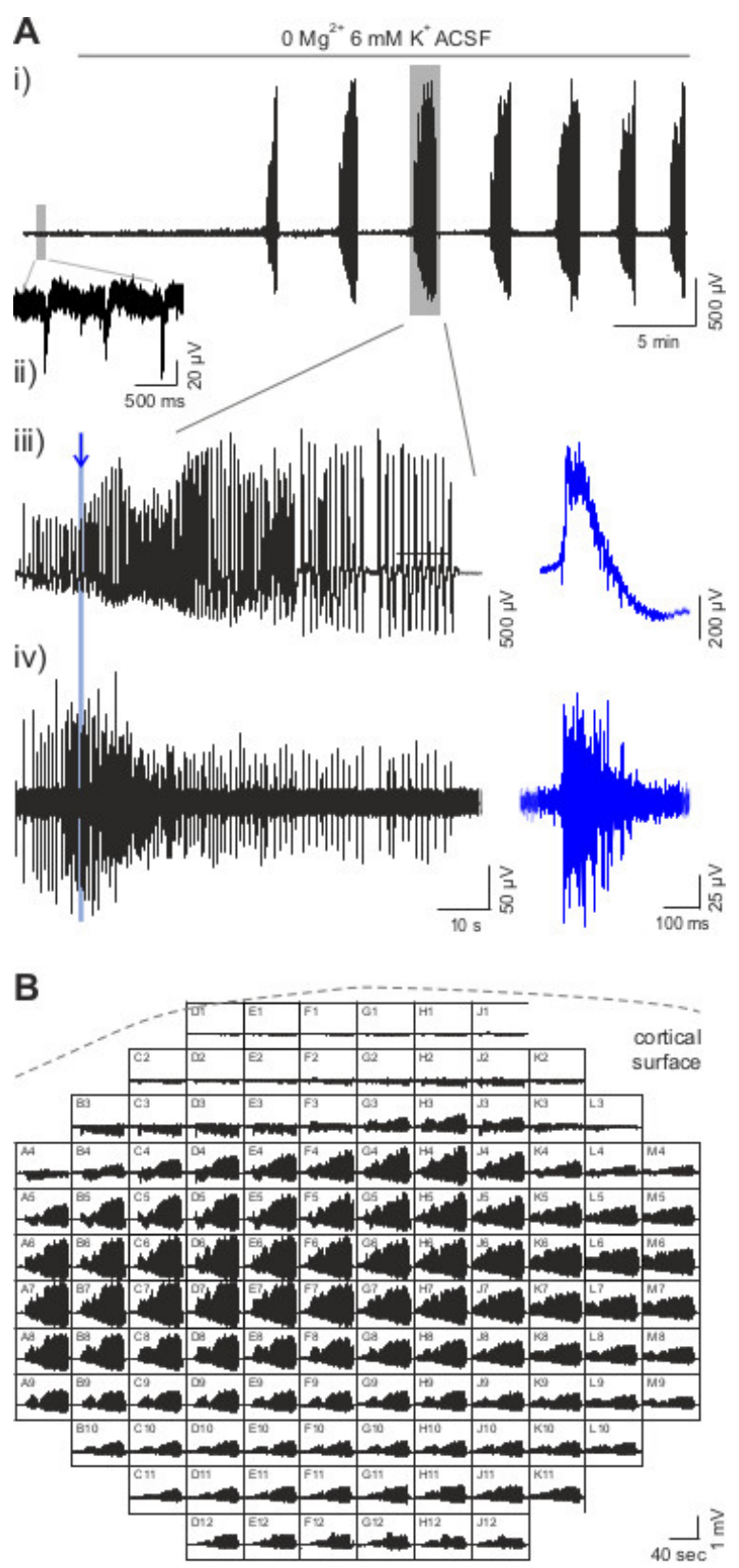

\section{Figure 3}

Figure 3: MEA recordings of epileptic seizures in human cortical slices. (A) A representative trace of human cortical slice activity recorded by a MEA electrode is shown in panel i. In presence of normal ACSF, it is possible to observe interictal-like spontaneous activity (small grey rectangle, zoomed in panel ii); then, when the perfusion with $0 \mathrm{Mg}^{2+} 6 \mathrm{mM} \mathrm{K}^{+} \mathrm{ACSF}$ has started, the first seizure appears after a $\sim 15$ min delay and is followed by other events at 2-3 min interval. Panel iii shows the seizure in the big grey rectangle in panel $\mathrm{i}$ with a zoomed scale. The blue trace illustrates a zoom of the activity, as indicated by the blue line and arrow. Panel iv) shows the data illustrated in panel iii) high pass filtered at $250 \mathrm{~Hz}$, which reveal multi-unit activity when zoomed (blue trace). (B) Representative recording of a seizure in presence of $0 \mathrm{Mg}^{2+} 6 \mathrm{mM} \mathrm{K}^{+}$ ACSF from all MEA electrodes. Each square represents an electrode of $12 \times 12$ MEA array and shows an 80 sec time window; the dotted line indicates the position of the cortical surface relatively to MEA array.

\section{Discussion}

Pharmacoresistant epilepsy is a rare condition, which can be explored in human tissue in vitro. This allows studying epileptic human cortex, which displays specific defects that are only partially reproduced in animal models. The method here described allows preparing and recording human postoperative tissues ex vivo with preserved cellular viability and networks so that they spontaneously produce epileptic activities.

Retaining similar activities than those recorded in vivo is crucial to study mechanisms of genesis of pathological activities. Further, such methods 
allow exploring human tissues and avoid non perfect animal models of the disease. However, studying human tissue requires synchronization between neurosurgeons and the experimental laboratory. Tissue transportation requires specific care not to be traumatic. In addition, both the quantity of specimen and tissue is limited. Finally, access to proper control tissues is the main concern. With this preparation, the post operative tissues spontaneously produce interictal-like discharges in normal $\mathrm{ACSF}^{7,8}$. Ictal-like events can also be elicited in modified, proconvulsive ACSF so that the mechanisms of seizure initiation and transition from the interictal state to seizures can be investigated.

Human tissue can be kept viable for up to $10 \mathrm{hr}$ in interface conditions. Slices were considered viable when multi-unit activity or field potentials were observed spontaneously and when such activities were evoked by increasing excitability through extracellular potassium increases and/or magnesium decrease. Although variability in activity occurs, likely reflecting differences in pathologies and cortical areas, we explored epileptogenic characteristics of a tissue only in healthy slices showing spontaneous interictal and evoked ictal discharges. In order to preserve tissue vitality and activity, an interface chamber is used to store the slices at $36-37^{\circ} \mathrm{C}$ for recovery before recording with the MEA system. Indeed, several groups have clearly demonstrated the advantages of interface based storage system compared to standard beaker storage and the importance of temperature for the preservation of network activity such as spontaneous sharp wave-ripples or cholinergic-induced oscillations $^{9,10}$. Interface storage of slices has been previously used to record epileptic activity from human hippocampal and subicular slices ${ }^{1,11}$ With the current MEA technique, after the recovery period from slicing in interface conditions, the network activity is recorded in submerged conditions, in presence of high flow rate $(5-6 \mathrm{ml} / \mathrm{min})$ at $37^{\circ} \mathrm{C}$, with the MEA system. The reduced diameter $(1.8 \mathrm{~cm})$ of MEA chip, which delimits a small volume chamber $(<1.5 \mathrm{ml})$, together with the increased flow rate, enhances oxygen supply of the slice, which has been demonstrated to be a critical factor for spontaneous and pharmacologically-induced network activities ${ }^{9,10}$. Further, the reduced amount of circulating ACSF facilitates pharmacological testing.

The slicing procedure is however a traumatism for the tissues ${ }^{12}$. Both neuronal architecture and chloride homeostasis appear to be perturbed at the tissue surface $(50 \mu \mathrm{m})$. The origin of the activities recorded by MEA chips, which sample the tissue mostly superficially without deep penetration, may arise from traumatized areas. However, our data show that the extracellular field potentials detected locally are recorded in most MEA electrodes and previous work show that they are integrated over a 100 to $200 \mu \mathrm{m}$ distance from the recording site ${ }^{13}$, suggesting that seizures recorded in our preparation are unlikely to be produced by traumatized areas. Further, in studies performed with tungsten electrodes enabling deep penetration, the epileptic activities recorded in human tissues are similar to the ones observed in epileptic patients ${ }^{1,7,8}$

Another limit of ex vivo tissue recording is the disruption of the connections between the different brain areas, thus restricting dynamic neuromodulations. This may explain why in such tissue no ictal-like event is spontaneously recorded, but requires to be triggered by ionic manipulation or pharmacological stimulation enhancing excitability. Accordingly, in this protocol, seizure-like events are induced by combining a change in extracellular $\mathrm{K}^{+}$from 3 to $6 \mathrm{mM}$ and a reduction of external $\mathrm{Mg}^{2+}$ from $1.3 \mathrm{mM}$ to $\mathrm{Mg}^{2+}$-free ACSF, in order to increase tissue excitability and remove $\mathrm{Mg}^{2+}$-dependent NMDA receptor block. Indeed, it has previously been demonstrated that epileptiform activity induced in human neocortical and hippocampal slices using $\mathrm{Mg}^{2+}$-free ACSF resembles the electrographic seizures recorded in vivo ${ }^{14}$. Besides, it has been shown that epileptiform discharges obtained in temporal lobe slices become resistant to clinically used anticonvulsants after prolonged exposure to $\mathrm{Mg}^{2+}$-free $\mathrm{ACSF}^{15,16}$, thus providing a model for investigating pharmacoresistant seizure-like events in vitro.

MEAs allow recording of both, field potentials and multi-unit activities consisting in neuronal action potentials ex vivo. Thus, MEAs are a powerful electrophysiological tool compared to EEGs, which explore field potentials generated by synchronous activities of neuronal ensembles in vivo, but do not give access to single neuron behaviors ${ }^{17}$. Although more recent microelectrodes can record in vivo multi-unit activities consisting in neuronal action potentials, they are invasive, so their use is mostly restricted to research purpose during intracranial recordings. In particular, MEAs recordings represent a technique of choice to study the spatio-temporal patterns of epileptic events, the mechanisms controlling seizure onset and propagation and the action of classic and new antiepileptic drugs. Remarkably, to unravel the cell types and the signaling basis of epileptic discharges, spike sorting techniques and pharmacological testing should be combined with MEA techniques. Although MEAs can give access to individual spikes, they do not provide information about synaptic and biophysical properties. In the future, other techniques should be coupled to MEA recordings in order to better sample cellular behavior, network activities and synaptic signaling. For instance, fluorescence imaging to unravel neurons or glial cells behavior, as well as ion dynamics, may be combined with MEAs recordings. Further post hoc histological analysis can also reveal specific alterations of cell types, proteins or receptors so that the location of the epileptic activities can be correlated with specific rearrangements of the nervous structure. The MEAs system can also be embedded in a patch-clamp set-up to correlate single cells or conductances with population activities. In the future, optogenetic tools could also be used, provided that human slices can be cultured in the long-term, as performed for organotypic slices, so that transfection or infection of specific cell types can be performed.

\section{Disclosures}

Sponsorship by Multichannel Systems was provided for the production of the video and open access publication.

\section{Acknowledgments}

This work was supported by grants from ANR (Programme Blanc Neurosciences), FRC (Fédération pour la Recherche sur le Cerveau), City of Paris (Programme Emergence), INSERM and La Pitié Salpêtrière Hospital (Translational research contract) to N.R., from Neuropôle de Recherche Francilien (NeRF) to E.D., from the Université Pierre et Marie Curie UPMC (Programme Convergence) and from the Institut du Cerveau et e la Moelle epiniere (Paris) to G.H.

\section{References}

1. Huberfeld, G., et al. Glutamatergic pre-ictal discharges emerge at the transition to seizure in human epilepsy. Nature Neuroscience. 14 (5), 627-634, doi:10.1038/nn.2790 (2011).

2. Kwan, P., \& Brodie, M. J. Early identification of refractory epilepsy. New England Journal of Medicine. 342 (5), $314-319$ (2000). 
3. Van Pelt, J., Corner, M. A., Wolters, P. S., Rutten, W. L. C., \& Ramakers, G. J. A. Longterm stability and developmental changes in spontaneous network burst firing patterns in dissociated rat cerebral cortex cell cultures on multielectrode arrays. Neuroscience Letters. 361 (1-3), 86-89, doi:10.1016/j.neulet.2003.12.062 (2004).

4. Sun, J.-J., \& Luhmann, H. J. Spatio-temporal dynamics of oscillatory network activity in the neonatal mouse cerebral cortex: Network oscillations in neonatal cerebral cortex. European Journal of Neuroscience. 26 (7), 1995-2004, doi:10.1111/j.1460-9568.2007.05819.x (2007).

5. Dossi, E., et al. Functional Regeneration of the ex-vivo Reconstructed Mesocorticolimbic Dopaminergic System. Cerebral cortex (New York, N.Y.: 1991). 23 (12), 2905-2922, doi:10.1093/cercor/bhs275 (2013).

6. Darbon, P., Scicluna, L., Tscherter, A., \& Streit, J. Mechanisms controlling bursting activity induced by disinhibition in spinal cord networks. The European journal of neuroscience. 15 (4), 671-683 (2002).

7. Cohen, I., et al. On the Origin of Interictal Activity in Human Temporal Lobe Epilepsy in Vitro. Science. 298 (5597), 1418-1421, doi:10.1126/ science.1076510 (2002).

8. Huberfeld, G., et al. Perturbed chloride homeostasis and GABAergic signaling in human temporal lobe epilepsy. The Journal of neuroscience. 27 (37), 9866-9873, doi:10.1523/JNEUROSCI.2761-07.2007 (2007).

9. Maier, N., Morris, G., Johenning, F. W., \& Schmitz, D. An Approach for Reliably Investigating Hippocampal Sharp Wave-Ripples In Vitro. PLoS ONE. 4 (9), e6925, doi:10.1371/journal.pone.0006925 (2009).

10. Hájos, N., et al. Maintaining network activity in submerged hippocampal slices: importance of oxygen supply. European Journal of Neuroscience. 29 (2), 319-327, doi:10.1111/j.1460-9568.2008.06577.x (2009).

11. Wittner, L., et al. The epileptic human hippocampal cornu ammonis 2 region generates spontaneous interictal-like activity in vitro. Brain. 132 (11), 3032-3046, doi:10.1093/brain/awp238 (2009).

12. Dzhala, V., Valeeva, G., Glykys, J., Khazipov, R., \& Staley, K. Traumatic alterations in GABA signaling disrupt hippocampal network activity in the developing brain. The Journal of neuroscience. 32 (12), 4017-4031, doi:10.1523/JNEUROSCI.5139-11.2012 (2012).

13. Menendez de la Prida, L., Huberfeld, G., Cohen, I., \& Miles, R. Threshold Behavior in the Initiation of Hippocampal Population Bursts. Neuron. 49 (1), 131-142, doi:10.1016/j.neuron.2005.10.034 (2006).

14. Avoli, M., Drapeau, C., Louvel, J., Pumain, R., Olivier, A., \& Villemure, J.-G. Epileptiform activity induced by low extracellular magnesium in the human cortex maintained in vitro. Annals of neurology. 30 (4), 589-596 (1991).

15. Heinemann, U., Dreier, J., Leschinger, A., Stabel, J., Draguhn, A., \& Zhang, C. Effects of anticonvulsant drugs on hippocampal neurons. Hippocampus. 4 (3), 291-296 (1994).

16. Li Zhang, C., Dreier, J. P., \& Heinemann, U. Paroxysmal epileptiform discharges in temporal lobe slices after prolonged exposure to low magnesium are resistant to clinically used anticonvulsants. Epilepsy research. 20 (2), 105-111 (1995).

17. Buzsáki, G., Anastassiou, C. A., \& Koch, C. The origin of extracellular fields and currents - EEG, ECoG, LFP and spikes. Nature Reviews Neuroscience. 13 (6), 407-420, doi:10.1038/nrn3241 (2012). 